\title{
Non-Uniqueness of Solutions of Percival's Euler-Lagrange Equation
}

\author{
John N. Mather* \\ Department of Mathematıcs, Princeton University, Princeton, NJ 08544, USA
}

\begin{abstract}
Percival $[5,6]$ introduced a Langrangian and an Euler-Lagrange equation for finding quasi-periodic orbits. In [3], we studied area preserving twist homeomorphisms of the annulus, using Percival's formalism. We showed that Percival's Lagrangian has a maximum on a suitable function space, and that a point where it takes its maximum is a solution of Percival's EulerLagrange equation. Moreover, in the rigorous interpretation of Percival's formalism which we gave in [3], the solutions of Percival's Euler-Lagrange equation correspond bijectively to a certain class of minimal sets. (We will prove this in Sect. 2.) In [4], we showed that Percival's Lagrangian takes its maximum at only one point. In this paper, we show that there exist $C^{\infty}$ area preserving twist diffeomorphisms of the annulus, for which there exists at least one solution of Percival's Euler-Lagrange equation where Percival's Lagrangian does not take its maximum. In other words, solutions of Percival's Euler-Lagrange equation need not be unique.
\end{abstract}

\section{Statement of the Results}

In this section, we recall basic notations, terminology, and results from [3] and [4], and state the theorem which we will prove in this paper.

We will denote by $\mathscr{F}$ the class of homeomorphisms which was considered in [3]. This is defined as follows.

We set $A=\left\{(x, y) \in \mathbb{R}^{2}: 0 \leqq y \leqq 1\right\}$. We let $T: A \rightarrow A$ be the translation $T(x, y)$ $=(x+1, y)$. We let $\mathscr{F}$ be the set of homeomorphisms of $A$ which satisfy the following two conditions:

1) $f$ is area preserving, orientation preserving, boundary component preserving, and $f T=T f$.

2) (positive monotone twist condition) $f(x, y)_{1}>f(x, z)_{1}$ if $y>z$. Here, $p_{1}$ denotes the first coordinate of $p$, if $p \in A=\mathbb{R} \times[0,1]$.

* Supported by NSF Grant No. MCS 79-02017 
If $r$ is a positive integer or $\infty$, we let $\mathscr{F}^{r}$ denote the set of all $C^{r}$ diffeomorphisms in $\mathscr{F}$ which satisfy

$$
\frac{\partial\left(f(x, y)_{1}\right)}{\partial x}>0
$$

Let $f \in \mathscr{F}$. Then $f$ maps each boundary component of $A$ onto itself and is orientation preserving on it. Let $f_{i}=f \mid \mathbb{R} \times i, i=0,1$. Since $f T=T f$, the Poincaré rotation numbers

are defined.

$$
\varrho\left(f_{i}\right)=\lim _{n \rightarrow \pm \infty} \frac{f_{i}^{n}(x)}{n}, \quad i=0,1,
$$

Let $B=B_{f}=\left\{\left(x, x^{\prime}\right) \in \mathbb{R}^{2}: f_{0}(x) \leqq x^{\prime} \leqq f_{1}(x)\right\}$. The conditions which we imposed on $f$ imply that it is defined by what is known in classical mechanics as a generating function. A generating function is a continuous, real valued function $h=h_{f}$ on $B$, which is continuously differentiable on the interior of $B$, such that

$$
f(x, y)=\left(x^{\prime}, y^{\prime}\right) \Leftrightarrow\left\{\begin{array}{l}
y=h_{1}\left(x, x^{\prime}\right) \\
y^{\prime}=-h_{2}\left(x, x^{\prime}\right),
\end{array}\right.
$$

where $h_{1}\left(x, x^{\prime}\right)=\partial h\left(x, x^{\prime}\right) / \partial x$ and $h_{2}\left(x, x^{\prime}\right)=\partial h\left(x, x^{\prime}\right) / \partial x^{\prime}$. Moreover, $h_{1}$ and $h_{2}$ extend continuously to the boundary of $B$. The function $h$ is uniquely determined up to an additive constant. The existence of such a function $h$ is well known in classical mechanics. Note that $h\left(x+1, x^{\prime}+1\right)=h\left(x, x^{\prime}\right)$. For slightly more detail in the construction of $h$, see [3].

The main object of our study is not $f$, but the mapping $\bar{f}$ of the annulus $A / T$ into itself induced by $f$. However, it is easier to state our results in terms of $f$.

We let $Y_{\omega}=Y_{f, \omega}$ denote the set of weakly order preserving $\phi: \mathbb{R} \rightarrow \mathbb{R}$ such that

$$
\phi(t+1)=\phi(t)+1, \quad f_{0}(\phi(t)) \leqq \phi(t+\omega) \leqq f_{1}(\phi(t)),
$$

and $\phi$ is continuous from the left. In [3], we proved:

Theorem. Suppose $\varrho\left(f_{0}\right) \leqq \omega \leqq \varrho\left(f_{1}\right)$. Then there exists $\phi \in Y_{\omega}$ such that

$$
f(\phi(t), \eta(t))=(\phi(t+\omega), \eta(t+\omega)),
$$

where $\eta(t)=h_{1}(\phi(t), \phi(t+\omega))$.

When $\omega$ is irrational, there exists a minimal set $\Sigma_{\phi}$ of the homeomorphism $\bar{f}$ associated to $\phi$. Explicitly, $\Sigma_{\phi}=M_{\phi} / T$, where $M_{\phi}$ is the closure of the set of $(\phi(t), \eta(t))$, where $t$ ranges over all points of continuity of $\phi$.

The existence of $\Sigma_{\phi}$ is the main significance of the above theorem. When $f$ admits an invariant circle of rotation number $\omega$, we have that $\Sigma_{\phi}$ is the unique minimal set in that circle. (This minimal set may be a Cantor set or the whole circle.) Otherwise, $\Sigma_{\phi}$ is an invariant Cantor set.

It is convenient to introduce the quantity $V=V_{f, \omega}$, defined by

$$
V_{\omega}(\phi)(t)=h_{2}(\phi(t-\omega), \phi(t))+h_{1}(\phi(t), \phi(t+\omega)) .
$$

In this notation, Percival's Euler-Lagrange equation is $V_{\omega}(\phi)=0$. For $\phi \in Y_{\omega}$

$$
f(\phi(t), \eta(t))=(\phi(t+\omega), \eta(t+\omega)), \quad t \in \mathbb{R},
$$

where $\eta(t)=h_{1}(\phi(t), \phi(t+\omega))$ is equivalent to $V_{c,}(\phi)=0$. This means that the above theorem is equivalent to the assertion that Percival's Euler-Lagrange equation has a solution. 
This motivates the following discussion of Percival's Euler-Lagrange equation.

Let $T_{a}$ denote the translation by $a$. In other words, $T_{a}(t)=t+a$. Obviously, if $\phi$ is a solution of Percival's Euler-Lagrange equation, then so is $\phi T_{a}$. Moreover, if $\phi^{\prime}=\phi T_{a}$, then $\Sigma_{\phi}=\Sigma_{\phi^{\prime}}$. This leads us to consider the quotient set $X_{\omega}=X_{f, \omega}=Y_{\omega} / \sim$, where $\sim$ is the equivalence relation defined by $\phi \sim \phi^{\prime} \Leftrightarrow \exists a$, $\phi^{\prime}=\phi T_{a}$. (Note that this is different from the $X_{\omega}$ defined in [3].)

In [3], we defined $F_{\omega}(\phi)=F_{f, \omega}(\phi)=\int_{0}^{1} h(\phi(t), \phi(t+\omega)) d t$. This is Percival's Lagrangian. We have $F_{\omega}\left(\phi T_{a}\right)=F_{\omega}(\phi)$. Hence, $F_{\omega}$ induces a function on $X_{\omega}$, which we also denote by $F_{\omega}$ (or $F_{f, \omega}$ ). In $\left[3\right.$, Sect. 4], we defined a metric $d$ on $Y_{\omega}$. It is obvious from the definition of $d$ that $d\left(\phi T_{a}, \phi^{\prime} T_{a}\right)=d\left(\phi, \phi^{\prime}\right)$. Hence, we may define a metric on $X_{\omega}$ by

$$
d\left(\bar{\phi}, \bar{\phi}^{\prime}\right)=\inf \left\{d\left(\phi, \phi^{\prime}\right): \phi \in \bar{\phi}, \phi^{\prime} \in \bar{\phi}^{\prime}\right\} .
$$

Provided with this metric, $X_{\omega}$ is compact. This follows from [3, Sect. 5]: the space which we denoted $X_{\omega}$ there maps surjectively and continuously to the space which we denote $X_{\omega}$ in this paper.

Moreover, by $\left[3\right.$, Sect. 6], $F_{\omega}: Y_{\omega} \rightarrow \mathbb{R}$ is continuous, so $F_{\omega}: X_{\omega} \rightarrow \mathbb{R}$ is continuous, since $X_{\omega}$ has the quotient topology. Since $F_{\omega}$ is a continuous function on a compact space, it takes its maximum at some point. Moreover, when $\omega$ is irrational, $F_{\omega}$ takes its maximum at only one point [4]. In [3], we showed that Percival's Euler-Lagrange equation is satisfied at a point where Percival's Lagrangian takes its maximum.

This may seem obvious, in view of the fact that $V_{\omega}(\phi)$ is the first variation of $F_{\omega}(\phi)$ in some formal sense. However, it is not obvious. Indeed, a point where Percival's Lagrangian takes its minimum is rarely (if ever) a solution of Percival's Euler-Lagrange equation. The fact that Percival's Euler-Lagrange equation is satisfied at a point where Percival's Lagrangian takes its maximum was the main step in the proof of the existence theorem in [3]. We believe that the methods of [3] are new; they don't fit into any standard framework of the calculus of variations. However, they are closely related to the ideas explained by G. D. Birkhoff in [1] for finding periodic orbits in the billiard ball problem by maximizing the perimeter of a polygon. It is obvious that one cannot find a periodic orbit of the billiard ball problem by minimizing the perimeter. The fact that Percival's Euler-Lagrange equation is satisfied at a point where Percival's Lagrangian takes its maximum, but not a point where it takes its minimum, closely parallels Birkhoff's theory of periodic orbits in the billiard ball problem.

In this paper, we show that Percival's Euler-Lagrange equation may have solutions where Percival's Lagrangian does not take its maximum. Specifically, we will prove:

Theorem. Let $\omega$ be an irrational number. There exists $f \in \mathscr{F}^{\infty}$ such that $\varrho\left(f_{0}\right)<\omega<\varrho\left(f_{1}\right)$ and $X_{f, \omega}$ contains more than one solution of Percival's EulerLagrange equation.

Note that there is some notational difficulty in saying exactly what Percival's Euler-Lagrange equation is for $\bar{\phi} \in X_{f, \omega}$. This is because

$$
V_{f, \omega}\left(\phi \circ T_{a}\right)=V_{f, \omega}(\phi) \circ T_{a} .
$$


Thus, $V_{f, \omega}(\bar{\phi})$ can only be defined as an equivalence class of functions, where two functions are equivalent if they differ only by composition with a translation on the right. However, the meaning of $V_{f, \omega}(\bar{\phi})=0$ is clear, since if one of the functions in this class satisfies this equation, then they all do.

At first sight, the theorem of this paper appears to contradict results proved in [3] and [4]. We have that $V_{f, \omega}$ is the first variation of $F_{f, \omega}$ in some formal sense. On the other hand, we proved [4] that when $\omega$ is irrational, $F_{f, \omega}$ is a strictly concave function on $X_{f, \omega}$, with respect to a suitable affine structure on $X_{f, \omega}$. Since the first derivative of a strictly concave function can vanish only where the function takes its maximum, there is an apparent contradiction. But it is not real.

Roughly speaking, the reason that there is no contradiction is that the affine structure on $X_{f, \omega}$ defines a different "differentiable structure" from the "differentiable structure" we used to define the first variation. Here, we use the term "differentiable structure" loosely. We do not attempt to formalize it. Since we do not use the notion of "differentiable structure" in any of our proofs, it is not necessary for us to formalize it.

To be more precise, we used certain test curves in [3] to compute the first variation. But, straight line segments with respect to the affine structure are not test curves of the type considered in [3]. In fact, $F_{\omega}$ is not necessarily even differentiable on straight line segments with respect to the affine structure. Moreover, to the extent which we can define a first variation using straight line segments as test curves, it seems that we get something different from $V_{\omega}(\phi)$. So, there is no contradiction.

\section{The Bijective Correspondence between Certain Minimal Sets and Solutions of Percival's Euler-Lagrange Equation}

Let $f \in \mathscr{F}$ and let $\bar{f}: A / T \rightarrow A / T$ denote the induced homeomorphism. Let $\omega$ be an irrational number satisfying $\varrho\left(f_{0}\right)<\omega<\varrho\left(f_{1}\right)$. In Sect. 1 , we reviewed how a solution $\phi \in Y_{f, \omega}$ of Percival's Euler-Lagrange equation gives rise to a minimal set $\Sigma_{\phi}$ of $\bar{f}$. The set $\Sigma_{\phi}$ depends only on the equivalence class $\bar{\phi}$ of $\phi$ in $X_{f, \omega}$. So, we can denote $\Sigma_{\phi}$ by $\Sigma_{\bar{\phi}}$.

In this section, we will prove that the mapping $\bar{\phi} \mapsto \Sigma_{\bar{\phi}}$ is a bijection of $\left\{\bar{\phi} \in X_{f, \omega}: V_{f, \omega}(\bar{\phi})=0\right\}$ onto a certain class $\mathscr{M}_{f, \omega}$ of minimal sets of $\bar{f}$. Closely related results are discussed in Katok [2].

Definition of $\mathscr{M}_{f, \omega}$. We let $\mathscr{M}_{f, \omega}$ be the collection of subsets $\Sigma$ of $A / T$ with the following four properties:

1) $\Sigma$ is minimal for $\bar{f}$, i.e., $\Sigma$ is closed and invariant, and it contains no closed and invariant sets other than itself and the empty set.

Since $A=\mathbb{R} \times[0,1]$, we have an obvious identification $A / T=(\mathbb{R} / \mathbb{Z}) \times[0,1]$. Let

$$
\pi_{1}: A / T=(\mathbb{R} / \mathbb{Z}) \times[0,1] \rightarrow \mathbb{R} / \mathbb{Z} \quad \text { and } \quad \pi_{1}: \mathbb{R} \times[0,1] \rightarrow \mathbb{R}
$$

denote the projections.

2) $\pi_{1} \mid \Sigma: \Sigma \rightarrow \mathbb{R} / \mathbb{Z}$ is injective.

Let $\pi_{T}: A \rightarrow A / T$ denote the projection. Let $M=\pi_{T}^{-1}(\Sigma)$. In view of 2 ), we have that $\pi_{1} \mid M: M \rightarrow \mathbb{R}$ is injective. The standard order on $\mathbb{R}$ induces an order on $\pi_{1}(M)$, and this defines an order on $M$, via $\pi_{1}^{-1}$. 
3) $f: M \rightarrow M$ is order preserving.

4) If $(x, y) \in M$, then $\lim _{n \rightarrow \pm \infty} \frac{f^{n}(x, y)_{1}}{n}=\omega$.

It is obvious that if $\phi \in X_{f,()}$ is a solution of Percival's Euler-Lagrange equation, then $\Sigma_{\phi} \in \mathscr{M}_{f, \omega}$. Moreover, if $\phi, \phi^{\prime} \in X_{f, \omega}$ are two different solutions of Percival's Euler-Lagrange equation, then $\Sigma_{\phi} \neq \Sigma_{\phi^{\prime}}$. The rest of this section will be devoted to a proof of the following result.

Proposition. If $\Sigma \in \mathscr{M}_{f, \omega}$, then there exists a solution $\phi \in X_{f, \omega}$ of Percival's EulerLagrange equation such that $\Sigma=\Sigma_{\phi}$.

Proof. Condition 4) in the definition of $\mathscr{M}_{f, \omega}$ implies that $\Sigma$ is infinite, since $\omega$ is irrational.

Since $\Sigma$ is compact and infinite, it contains non-isolated points. The set of all non-isolated points forms a closed and invariant set. Since $\Sigma$ is minimal for $\bar{f}$, it follows that $\Sigma$ contains no isolated points.

By a complementary interval of $\pi_{1}(\Sigma)$, we will mean a connected component of $\mathbb{R} / \mathbb{Z} \backslash \pi_{1}(\Sigma)$. Let $\Sigma^{\prime}$ be the quotient space of $\pi_{1}(\Sigma)$ obtained by identifying opposite endpoints of complementary intervals of $\pi_{1}(\Sigma)$. Since $\pi_{1} \mid \Sigma$ is injective, $\pi_{1}(\Sigma)$ is infinite and has no isolated points, just as is the case for $\Sigma$. Hence, $\Sigma^{\prime}$ is homeomorphic to the circle.

Condition 3 ) in the definition of $\mathscr{M}_{f, \omega}$ guarantees that $\bar{f}: \Sigma \rightarrow \Sigma$ respects the identifications in $\pi_{1}(\Sigma) \approx \Sigma$, which define $\Sigma^{\prime}$. Hence, we have an induced homeomorphism $f^{\prime}: \Sigma^{\prime} \rightarrow \Sigma^{\prime}$. Since $\Sigma$ is minimal for $\bar{f}$, we have that $\Sigma^{\prime}$ is minimal for $f^{\prime}$. It follows that $f^{\prime}$ is topologically conjugate to an irrational rotation. Condition 4) in the definition of $\mathscr{M}_{f, \omega}$ guarantees that the corresponding rotation number is $\equiv \omega(\bmod 1)$.

Let $p: \mathbb{R} / \mathbb{Z} \rightarrow \Sigma^{\prime}$ be the unique extension of the projection $\pi_{1}(\Sigma) \rightarrow \Sigma^{\prime}$ which is constant on each complementary interval of $\pi_{1}(\Sigma)$. Let $h: \Sigma^{\prime} \rightarrow \mathbb{R} / \mathbb{Z}$ be a homeomorphism which conjugates $f^{\prime}$ and the rotation through $\omega$. The mapping $h p: \mathbb{R} / \mathbb{Z} \rightarrow \mathbb{R} / \mathbb{Z}$ lifts to a continuous mapping $\psi: \mathbb{R} \rightarrow \mathbb{R}$ which is weakly order preserving and satisfies $\psi(x+1)=\psi(x)+1$.

The definition of $h$ implies

$$
h p\left(\pi_{1} \bar{f}(\bar{x}, y)\right)=h p(\bar{x})+\omega(\bmod 1), \quad(\bar{x}, y) \in \Sigma .
$$

As before, we let $M=\pi_{T}^{-1} \Sigma$. The above equation and condition 4) in the definition of $\mathscr{M}_{f, \omega}$ imply

$$
\psi\left(\pi_{1} f(x, y)\right)=\psi(x)+\omega, \quad(x, y) \in M .
$$

Since $f_{0}(x) \leqq \pi_{1} f(x, y) \leqq f_{1}(x)$, we then obtain

$$
\psi\left(f_{0}(x)\right) \leqq \psi(x)+\omega \leqq \psi\left(f_{1}(x)\right) .
$$

Let $\phi=I(\psi)$, where $I$ is as defined in [4]. In other words, $I$ is left-continuous, and

Then $\phi \in Y_{f, \omega}$.

$$
\left(x, x^{\prime}\right) \in \operatorname{graph} \phi \Leftrightarrow\left(x^{\prime}, x\right) \in \operatorname{graph} \psi
$$

Let $t$ be a point of continuity of $\phi$. Then $\phi(t) \in \pi_{1}(M)$ and is not the endpoint of a complementary interval of $\pi_{1}(M)$. Let $\eta(t)$ be such that $(\phi(t), \eta(t)) \in M$. Let $(x, y)$ 
$=(\phi(t), \eta(t))$ and $\left(x^{\prime}, y^{\prime}\right)=f(x, y)$. Since $x^{\prime} \in \pi_{1} M$ and is not an endpoint of a complementary interval of $\pi_{1} M$, we have that $\psi\left(x^{\prime}\right)$ is a point of continuity of $\phi$. Hence,

$$
x^{\prime}=\phi \psi\left(x^{\prime}\right)=\phi \psi\left(\pi_{1} f(x, y)\right)=\phi(\psi(x)+\omega)=\phi(t+\omega) .
$$

Since $\left(x^{\prime}, y^{\prime}\right) \in M$, it then follows from the definition of $\eta$ that $y^{\prime}=\eta(t+\omega)$. Since $f(x, y)=\left(x^{\prime}, y^{\prime}\right)$, we have $y=h_{1}\left(x, x^{\prime}\right)$. In other words,

$$
f(\phi(t), \eta(t))=(\phi(t+\omega), \eta(t+\omega))
$$

and $\eta(t)=h_{1}(\phi(t), \phi(t+\omega))$. It is easily seen that this implies that $\phi$ is a solution of Percival's Euler-Lagrange equation.

From the definition of $\phi$, it follows easily that the closure of the set of $\phi(t)$ such that $t$ is a point of continuity of $\phi$ is $\pi_{1} M$. Moreover, $(\phi(t), \eta(t)) \in M$ by the definition of $\eta(t)$. Hence $M=M_{\phi}$ and so $\Sigma=\Sigma_{\phi}$.

In view of the proposition and the discussion preceding it, $\phi \mapsto \Sigma_{\phi}$ is a bijection of the set of solutions of Percival's Euler-Lagrange equation in $X_{f, \omega}$ onto $\mathscr{M}_{f, \omega}$.

\section{Examples where Percival's Euler-Lagrange Equation has more than One Solution}

We wish to show that an example exists for every irrational number $\omega$. It is clearly enough to consider the case when $0<\omega<1$, since we may replace $f$ by $f T^{k}$. We will give a single example which works whenever $0<\omega<1$. In fact, in the example which we construct, there is a one parameter family of solutions, for each irrational $\omega$ in $[0,1]$.

The fact that there is a whole one parameter family of solutions and not just several solutions was suggested to me by Scott Shenker, who told me that numerically there are scads of solutions.

A slight modification of our construction gives families parametrized by several variables.

One parameter families of solutions can be defined for the mapping associated to the generating function

$$
h\left(x, x^{\prime}\right)=-\frac{\left(x^{\prime}-x\right)^{2}}{2}+k \sin 4 \pi x,
$$

whenever $k$ is large enough. The main feature of this generating function which permits the construction of a one parameter family of solutions is that $\sin 4 \pi x$ has two large humps in the unit interval. Likewise, if we replaced $\sin 4 \pi x$ with a periodic function of period 1 having $n$ large humps in the unit interval, we would get an $(n-1)$ parameter family of solutions. The function $\sin 2 \pi n x$ is an example of such a function.

However, the mapping associated to the above generating function does not map $A$ into itself. Since we wish to produce an example which maps $A$ into itself, we need to modify the above generating function. We will use a generating function of the form

$$
h\left(x, x^{\prime}\right)=-\frac{\left(x^{\prime}-x+1000\right)^{2}}{4002}-v\left(x^{\prime}-x\right)+\frac{u\left(x^{\prime}-x\right) \sin 4 \pi x}{8004} .
$$


Here, we take $u$ to be a $C^{\infty}$ real valued function of real variable satisfying $0 \leqq u \leqq 1$, $u(t)=1$ for $-1 \leqq t \leqq 2, u(t)=0$ for $t \leqq-2$ or $t \geqq 3, u(t)=u(1-t),\left|u^{\prime}\right|<3$, and $\left|u^{\prime \prime}\right|<10$. Such a function clearly exists. Set $C=\frac{3 \pi}{2001}+\frac{10}{8004}$. We take $v$ to be a $C^{\infty}$ function of a real variable satisfying $v(t)=0$ for $0 \leqq t \leqq 1, v^{\prime \prime}(t) \geqq 0$ everywhere, $v(t)=v(1-t)$,

$$
\begin{aligned}
& v^{\prime \prime}(t) \geqq C, \quad \text { if } \quad-2 \leqq t \leqq-1 \quad \text { or } \quad 2 \leqq t \leqq 3, \\
& v(t)=2 C\left(t-\frac{5}{2}\right) \quad \text { if } \quad 3 \leqq t, \\
& =-2 C\left(t+\frac{2}{3}\right) \text { if }-2 \geqq t .
\end{aligned}
$$

Such a function clearly exists.

Our conditions on $u$ and $v$ imply $h_{12} \geqq(2001)^{-1}$ everywhere, since

$$
h_{12}\left(x, x^{\prime}\right)=\frac{1}{2001}+v^{\prime \prime}\left(x^{\prime}-x\right)+\frac{\pi u^{\prime}\left(x^{\prime}-x\right) \cos 4 \pi x}{2001}-\frac{u^{\prime \prime}\left(x^{\prime}-x\right) \sin 4 \pi x}{8004} \text {. }
$$

We define $f$ by

$$
f(x, y)=\left(x^{\prime}, y^{\prime}\right) \Leftrightarrow\left\{\begin{array}{l}
y=\partial h\left(x, x^{\prime}\right) / \partial x \\
y^{\prime}=-\partial h\left(x, x^{\prime}\right) / \partial x^{\prime} .
\end{array}\right.
$$

We must show that this defines a mapping of $A$ into itself. In fact, $f$ is single valued and everywhere defined in $\mathbb{R}^{2}$, and $f(A)=A$. For, the equation $y=\frac{\partial h\left(x, x^{\prime}\right)}{\partial x}$ can be uniquely solved for $x^{\prime}$ as a function of $x$ and $y$, since $h_{12} \geqq(2001)^{-1}$ everywhere. This means that $f$ is single valued and everywhere defined on $\mathbb{R}^{2}$. Moreover, the equations $y=\frac{\partial h\left(x, x^{\prime}\right)}{x}, y^{\prime}=-\frac{\partial h\left(x, x^{\prime}\right)}{x^{\prime}}$ imply

$$
\begin{aligned}
& y^{\prime}=y=\frac{x^{\prime}-x+1000}{2001}+2 C, \quad \text { if } \quad x^{\prime}-x \geqq 3, \\
& y^{\prime}=y=\frac{x^{\prime}-x+1000}{2001}-2 C, \quad \text { if } \quad x^{\prime}-x \leqq-2 .
\end{aligned}
$$

Hence, $f(A)=A$. It is easily checked that $f \in \mathscr{F}^{\infty}$.

Next, we construct a one-parameter family of solutions of Percival's EulerLagrange equation for this $f$.

Let

$$
Y_{\omega}^{a}=\left\{\phi \in Y_{\omega, f}: \phi((0, a]) \subset\left[0, \frac{1}{4}\right] \text { and } \phi((a, 1]) \subset\left[\frac{1}{2}, \frac{3}{4}\right]\right\} .
$$

Let $\pi: Y_{\omega, f} \rightarrow X_{\omega, f}$ denote the projection. Let $X_{\omega}^{a}=\pi\left(Y_{\omega}^{a}\right)$. It is easily checked that $X_{\omega}^{a}$ is closed in $X^{\omega}$. Since $F_{\omega}: X_{\omega} \rightarrow \mathbb{R}$ is continuous and $X_{\omega}$ is compact, there exists $\bar{\phi}^{a} \in X_{\omega}^{a}$ which maximizes $F_{\omega} \mid X_{\omega}^{a}$, for any $a$ satisfying $0 \leqq a \leqq 1$.

Lemma 1. If $0 \leqq a \leqq 1$, then the element $\bar{\phi}^{a}$ of $X_{\omega}^{a}$ which maximizes $F_{\omega} \mid X_{\omega}^{a}$ is a solution of Percival's Euler-Lagrange equation.

For notational reasons, it is somewhat simpler to work in $Y_{\omega}^{a}$ than in $X_{\omega}^{a}$. Note that $\pi: Y_{\omega}^{a} \rightarrow X_{\omega}^{a}$ is a bijection. Let $\phi^{a}=\left(\pi \mid Y_{\omega}^{a}\right)^{-1}\left(\bar{\phi}^{a}\right)$. We will need the following result, in order to prove Lemma 1.

Lemma 2. $\phi^{a}((0, a]) \subset[0.01,0.24]$ and $\phi^{a}((a, 1]) \subset[0.51,0.74]$. 
Proof. Set $\phi=\phi^{a}$. Since $0<\omega<1,0 \leqq \phi(t+\omega)-\phi(t) \leqq 1$. Hence $u(\phi(t+\omega)-\phi(t))=1$ and $v(\phi(t+\omega)-\phi(t))=0$. Hence

Define

$$
h(\phi(t), \phi(t+\omega))=\frac{(\phi(t+\omega)-\phi(t)+1000)^{2}}{4002}+\frac{\sin 4 \pi \phi(t)}{8004} .
$$

$$
\begin{aligned}
\phi^{\prime}(t)=0.01 & \text { if } & 0 \leqq \phi(t) \leqq 0.01 \\
=\phi(t), & \text { if } & 0.01 \leqq \phi(t) \leqq 0.24 \\
=0.24, & \text { if } & 0.24 \leqq \phi(t) \leqq 0.25 \\
=0.51, & \text { if } & 0.5 \leqq \phi(t) \leqq 0.51 \\
=\phi(t), & \text { if } & 0.51 \leqq \phi(t) \leqq 0.74 \\
=0.74, & \text { if } & 0.74 \leqq \phi(t) \leqq 0.75,
\end{aligned}
$$

and require that $\phi^{\prime}(t+1)=\phi^{\prime}(t)+1$. In view of the fact that $\phi \in Y^{a}$, we have that $\phi^{\prime}$ is well defined and $\phi^{\prime} \in Y^{a}$. A calculus excercise shows that

$$
h\left(\phi^{\prime}(t), \phi^{\prime}(t+\omega)\right) \geqq h(\phi(t), \phi(t+\omega)),
$$

with equality if and only if $\phi^{\prime}(t)=\phi(t)$ and $\phi^{\prime}(t+\omega)=\phi(t+\omega)$. Hence,

$$
F_{\omega}\left(\phi^{\prime}\right) \geqq F_{\omega}(\phi),
$$

with equality if and only if $\phi^{\prime}=\phi$. Since we have assumed that $\phi$ maximizes $F_{\omega} \mid Y_{\omega}^{a}$ we must have $\phi^{\prime}=\phi$, which implies the conclusion of Lemma 2.

Proof of Lemma 1. In [3, Sect. 10], we showed that if $F_{\omega}$ takes its maximum at $\phi \in Y_{\omega}$, then $\phi$ satisfies Percival's Euler-Lagrange equation. The reasoning we gave there actually shows somewhat more: Suppose $\phi \in F_{\omega}$ and $\phi$ does not satisfy Percival's Euler-Lagrange equation. We constructed a one-parameter family $\phi_{s}$ of elements $Y_{\omega}$, defined for $a \leqq s \leqq b$, where $a \leqq 0 \leqq b$ and $a<b$. (In [3], this family was denoted by $\phi_{s}, \psi_{s}$, or $\xi_{s}$, depending on the case.) For each fixed $t$, we had that $\phi_{s}(t)$ depended twice continuously differentiably on $s$, the functions

$$
(s, t) \mapsto \frac{\partial \phi_{s}(t)}{\partial s} \text { and } \quad(s, t) \mapsto \frac{\partial^{2} \phi_{s}(t)}{\partial s^{2}}
$$

are uniformly bounded and measurable in $[a, b] \times \mathbb{R}$, and $\left.\frac{d}{d s} F_{\omega}\left(\phi_{s}\right)\right|_{s=0} \neq 0$. Moreover, in the case $a=0$, we had $\left.\frac{d}{d s} F_{\omega}\left(\phi_{s}\right)\right|_{s=0}>0$, and, in the case $b=0$, we had $\left.\frac{d}{d s} F_{\omega}\left(\phi_{s}\right)\right|_{s=0}<0$.

Suppose $\phi^{a}$ does not satisfy Percival's Euler-Lagrange equation and take $\phi=\phi^{a}$. Then we have a one parameter family $\phi_{s}$, as above. Since $(s, t) \mapsto \frac{\partial \phi_{s}(t)}{\partial s}$ is uniformly bounded on $[a, b] \times \mathbb{R}$, it follows from Lemma 2 that $\phi_{s} \in Y^{a}$ for all $s$ with $|s|$ sufficiently small. But $F_{\omega}\left(\phi_{s}\right)>F_{\omega}(\phi)$ for all such $s$ satisfying $s>0$ or for all such $s$ satisfying $s<0$, according to the sign of $\left.\frac{d}{d s} F_{\omega}\left(\phi_{s}\right)\right|_{s=0}$. This contradicts the assumption that $\phi^{a}$ maximizes $F_{\omega} \mid Y_{\omega}^{a}$.

This contradiction shows that $\phi^{a}$ satisfies Percival's Euler-Lagrange equation. 


\section{Some Other Invariant Sets}

A slight modification of the construction in Sect. 3 gives an example of a $\Sigma \subset A / T$ which satisfies conditions 1,2 , and 4 , but not condition 3 in the definition of $\mathscr{M}_{f, \omega}$.

Let $T_{1 / 2}: A \rightarrow A$ be defined by $T_{1 / 2}(x, y)=\left(x+\frac{1}{2}, y\right)$. The mapping $f$ defined in the previous section obviously commutes with $T_{1 / 2}$, so it induces a mapping $\overline{\bar{f}}$ on $A / T_{1 / 2}$, as well as the mapping $\bar{f}$ on $A / T$. The obvious projection of $A / T$ onto $A / T_{1 / 2}$ is a 2 -fold covering mapping. Take $\phi=\phi^{0}$ (or $\phi^{1}$ ) in the notation of the previous section. Let $\Sigma \subset A / T_{1 / 2}$ denote the image of $\Sigma_{\phi} \subset A / T$ under the obvious projection.

It is easily seen that this $\Sigma$ satisfies conditions 1,2 , and 4 , but not condition 3 in the definition of $\mathscr{M}_{f, \omega}$, where $A / T$ is replaced by $A / T_{1 / 2}$.

\section{Continuous Dependence of $\phi^{a}$ on $a$}

It is easily seen that

$$
I\left(Y_{\omega}^{a}\right)=\left\{\psi \in \bar{Y}_{\omega}: \psi\left(\left[-\frac{1}{4}, 0\right]\right)=0, \psi\left(\left[\frac{1}{4}, \frac{1}{2}\right]\right)=a\right\},
$$

in the notation of [4]. Hence $I\left(Y_{\omega}^{a}\right)$ is a convex set. Thus, $I\left(\phi^{a}\right)$ maximizes the continuous, strictly concave function $F_{\omega} I$ over the convex, compact set $I\left(Y_{\omega}^{a}\right)$. It is easily seen that for any $a, a^{\prime} \in[0,1]$ and any $\phi \in Y_{\omega}^{a}$, there exists $\phi^{\prime} \in Y_{\omega}^{a^{\prime}}$ such that $d\left(\phi^{\prime}, \phi\right) \leqq\left|a^{\prime}-a\right|$. From this and the strict concavity of $F_{\omega} I$, the continuous dependence of the maximizing element $\phi^{a}$ on $a$ follows easily.

\section{References}

1. Birkhoff, G.D.: On the periodic motions of dynamical systems. Acta Math. 50, 359--379 (1927). Reprinted in: Collected Mathematical Papers, Vol. II, pp. 333-353, New York: American Math. Soc. 1950

2. Katok, A.: Some remarks on Birkhoff and Mather twist map theorems. Ergodıc theory and dynamical systems (to appear)

3. Mather, J.N. : Existence of quasi-periodic orbits for twist homeomorphisms of the annulus. Topology (to appear)

4. Mather, J.N.: Concavity of the Lagrangıan for quasi-periodic orbits. Comment. Math. Helv. (to appear)

5. Percival, I.C.: Variational principles for invariant Tori and Cantori. In: Symposium on Nonlinear Dynamics and Beam-Beam Interactions, American Inst. of Physics, Conf. Proc. No. 57, ed. M. Month and J.C. Herrara, pp. 302-310. New York: American Institute of Physics 1980

6. Percival, I.C.: J. Phys. A : Math. Nucl. Gen. 12, L57 (1979)

Communicated by T. Spencer

Received May 22, 1982 
